\title{
Implementación de un programa disciplinario mediado por las TIC para mejorar la convivencia escolar ${ }^{1}$
}

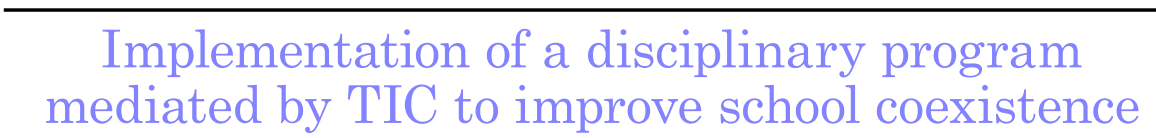

DOI: http://dx.doi.org/10.17981/cultedusoc.9.3.2018.31

\author{
Artículo de investigación. Fecha de recepción: 15/06/2018. Fecha de aceptación: 27/11/2018 \\ Omar Gastelbondo²; \\ Ninfa Argas; Idelfonso Ortega y Adira Ojeda-Moreno3 \\ Institución Educativa Departamental San José de Pueblo Viejo (Colombia) \\ josemora07@hotmail.com
}

Para citar este artículo:

Gastelbondo, O., Argas, N., Ortega, I. y Ojeda-Moreno, A. (2018). Implementación de un programa disciplinario mediado por las TIC para mejorar la convivencia escolar. Cultura. Educación y Sociedad 9(3), 273-280. DOI: http://dx.doi.org/10.17981/cultedusoc.9.3.2018.31

\section{Resumen}

Actualmente las instituciones educativas, están realizando acciones pedagógicas que propendan por la mejora de la convivencia escolar, puesto que la escuela debe ser un espacio que genere la participación estudiantil a través de una gestión asertiva de los conflictos. Por lo anterior el presente estudio tuvo como objetivo implementar un programa disciplinario mediado por las TIC para mejorar la convivencia escolar, La metodología es de tipo cualitativa, de alcance descriptivo, guiada bajo los planteamientos de la investigación como estrategia pedagógica IEP, utilizando como técnicas de recolección la observación y las bitácoras, la unidad de análisis estuvo conformada por 178 estudiantes de la Institución Educativa San José de Pueblo Viejo, del Departamento de Magdalena. Los resultados generaron comités disciplinarios, con reglamentos, así mismo se evidenció un clima en armonía en el desarrollo de las actividades, se siguen ruta de manejo de conflictos, y el trabajo mancomunado entre el maestro y el estudiante de acuerdo a los intereses.

Palabras clave: programa disciplinario, tecnologías de la información y la comunicación, convivencia escolar.

\section{Abstract}

Currently, educational institutions are carrying out pedagogical actions that favor the improvement of school coexistence, since the school must be a space that generates student participation through assertive management of conflicts. Therefore, the objective of this study was to implement a disciplinary program mediated by ICT to improve school coexistence. The methodology is of a qualitative nature, of descriptive scope, guided by the research approaches as an IEP pedagogical strategy, using collecting the observation and the logs, the analysis unit was made up of 178 students from the San José Educational Institution of Pueblo Viejo, in the Department of Magdalena. The results generated disciplinary committees, with regulations, likewise a climate in harmony in the development of the activities was evidenced, the conflict management route is followed, and the joint work between the teacher and the student according to the interests.

Keywords: disciplinary program, information and communication technologies, school coexistence.

1 Este artículo ha sido derivado del Programa de Fortalecimiento de la Cultura Ciudadana y Democrática CT+I a través de la IEP apoyada en TIC en el Departamento de Magdalena: CICLON

2 Docentes de la Institución Educativa Departamental San José de Pueblo Viejo y líder del grupo de investigación: Grupo de investigación "Los Innovadores".

3 Docentes de la Institución Educativa Departamental San José de Pueblo Viejo y miembros del Grupo de investigación "Los Innovadores".

- The author; licensee Universidad de la Costa - CUC.

Cultura, Educación y Sociedad vol. 9 no. 3, pp. 273-280. Diciembre, 2018

Barranquilla. ISSN 2389-7724 Online 


\section{Introducción}

La indisciplina ha sido uno de los temas que ha cobrado gran importancia en el sector educativo, pues evita que se lleven a cabo las actividades escolares de manera correcta, impidiendo el desarrollo de competencias por parte de los estudiantes debido a la falta de atención debida a la ausencia de disciplina. En la Institución Educativa San José de Pueblo Viejo, como en otras Instituciones Educativas, se presentan muy a menudo situaciones de indisciplina que alteran la convivencia escolar, en el aula de clase, dificultando el desarrollo de las mismas, por lo cual el presente estudio buscó Implementar un programa disciplinario mediado por las tecnologías de la información y la comunicación (TIC) para mejorar la convivencia escolar.

Según Aquino, (1996). Los actos de indisciplina son "todas las acciones, palabras, actitudes, gestos y reacciones que contrarían las normas disciplinarias vigentes en un centro de enseñanza, o que representan atentados contra la moral, la autoridad, el orden, el espíritu y las tradiciones de la institución”. P.p 13.Los actos de indisciplina atentan contra la ética e integralidad de cualquier institución educativa, y distorsionan los objetivos, que resultan la razón sustancial de éstas. (Garcia, 2011). Es por esta razón que la intención de los colegios es mitigar todas las acciones de indisciplina manifestadas por los estudiantes bajo comportamientos socialmente alarmantes, en torno a agresiones, peleas, insultos etc. (Dussel, 2005).

Son muchos los factores que pueden afectar o alterar la convivencia en una comunidad educativa; y algunas de las razones más comunes se relacionan con el grado de inmadurez de los estudiantes e introyección de normas y las razones que justifican su razón de ser. De la misma manera, es posible asumir que los adolescentes aun no están en condiciones de calcular las conse- cuencias de sus palabras, actos, actitudes y; establecer un control mental sobre las conductas esperadas en los procesos de interacción social (Hernández, 2007).

Es el tiempo, aclaración y corrección de hechos inapropiados, los entornos eductaivos y de socialización de reglas, normas y valores; los que hacen que poco a poco el adolecente pueda instaurar un sistema de estrategias que le permitan el control reflexivo, para generar conductas disciplinadas y reguladas.

Es deber de las escuelas entonces, estimular, favorecer y propender por la generación de espacios de intercambio para la progresiva maduración de los escolares, sin perjudicar la evolución normal y sana de su personalidad (Narodowski, 2012).

Lo anterior en el entendido de que la humanidad debe ser formada como sociedad de líderes éticos e integrales que puedan emplear habilidades para consensuar entre miembros de grupos y dirigir acciones por y para un bien común de países, organismos, instituciones entre otros. (Kadi y Acevedo, 2014)

En educación es importante, además, mencionar la disciplina y la indisciplina. La primera hace alusión a todas las normas reglas y procedimientos que se aplican al interior de las aulas de clases con el propósito de lograr los objetivos planteados. (Furlán, 2005). La segunda, es todo lo opuesto a la primera en donde existe la ausencia de valores y respeto a las normas.

En los centros educativos se deben enscender las alarmas en cuanto a la disciplina, proporcionando acciones que promuevan la seguridad, la responsabilidad y el respeto entre los actores, no dejando de lado que resulta un problema complejo. (Furlán, 2000).

El reglamento que asuman los centros escolares (propio o impropio), regula las acciones y las relaciones entre sus miembros; y la disciplina dependerá del nivel de relaciones establecidas en el aula, el interés 
y motivación que provoque el docente en cada uno de los estudiantes y en el grupo en general; y del tipo de procesos de comunicación establecidos entre ellos (Fierro, 2005). Esta acción debe estar abocada a la búsqueda de un número importante de instrumentos que garanticen suplir las necesidades disciplinarias (González, Moreno y Rodríguez, 2015).

Para muchas personas, un maestro competente es quien mantiene únicamente el orden y disciplina en las aulas de clase (Mares, 2009). Sin embargo, es necesario también tener en cuenta las restricciones que deben establecerse y los factores que alteran la conducta de los estudiantes; entre las que podemos identificar:

- El YO: Los nuevos modelos de familia e interacción social de la actualidad, han conducido a una pérdida de valores, estancamiento del desarrollo emocional y sentimientos de soledad, falta de confianza y seguridad en los niños, que los ha ubicado en una posición egoísta, en la que lo único que importa en la satisfacción de las propias necesidades (Ortega, 2006).

- Personalidad y carácter: Estos aspectos son fundamentales para la instauración de la disciplina. Es común observar niños y jóvenes con distintos temperamentos y caracteres; unos más difíciles de manejar que otros. Por tal razón, es necesario que en las aulas de clase se genere espacios diversos de interacción y aprendizaje, que motiven a los estudiantes a aprender y que permitan que se mantengan atentos a todas la actividades (Ortega, 2016).

- Liderazgo y poder: Uno de los factores que afecta la conducta de los estudiantes es la falta de poder y liderazgo ante determinadas situaciones, puesto que al no ser tenidos en cuenta, manifiestan conductas disruptivas en voz de protesta, que atentan con las dinámicas cotidianas (Peiró, 2008).
- Ambiente familiar: En la actualidad es común observar familias con pocos miembros, con ambos padres ocupados y niños bajo la crianza y cuidado de abuelos $u$ otras personas. Tal situación conlleva a una seria preocupación sobre las reglas y valores, puesto que el concepto de sí mismo es fundamentado en el hogar. Si un niño no establece esos procesos inciales de interacción en el hogar, que dan cuenta de la formación de normas, valores y moral; el adulto no tendrá la suficiente madurez y seguridad para enfrentar los desafío de la cotidianidad a la que se expondrá, por tal razón, es importante generar en ellos un ambiente familiar estable y seguro (Del Rey, 2009).

- Violencia social (Gotzens, et al., 2015). En lo relacionado con la violencia social, es posible identificar que los niños y jóvenes resuelven los problemas con violencia, observan las conductas violentas de sus padres y cuidadores y; ellos mismos son víctimas de violencia y malos tratos, lo que los hace insensibles a ella.

Adicionalmente, es común observar cómo la violencia es asumida como algo natural; afectando a segmentos sociales numerosos; lo cual conduce inevitablemente al aumento de la delincuencia, deficiencia formativa en las escuelas y muchas crisis sociales, políticas e individuales de la sociedad. (Gil, 2004).

- Medios de comunicación: Los incidentes violentos en las escuelas y en la cotidianidad en general, la mayoría de las veces son emitidos a través de los medios de comunicación. Es posible observar cómo programas, películas, series y noticieros, muestras imágenes y sucesos violentos, que sin duda promueven la cultura del maltrato en la sociedad. Incluso los programas infantiles hoy en día incluyen contenidos fuertes de violencia y sexo y no son censurados por las comisiones reguladoras de radio y televisión (Rodríguez, 2007). 
- Establecimiento de límites: Si los reglamentos o normas estudiantiles no son definidas tanto por los directivos, como por los maestros y estudiantes, será más difícil el establecimiento de límites y la claridad en los mismos, lo cual sin duda traería dificultades en la convivencia de los actores de la comunidad estudiantil.

- Desarrollo de la inteligencia emocional: Es importante generar espacios en los que el estudiante pueda ser capaz de identificar y expresar sentimientos y emociones; puesto que las normas generalmente indican lo que se puede hacer, sin dar alternativas (Saucedo, 2012).

Con base en estos factores, los docentes pueden establecer consensos con los estudiantes, para definir en conjunto estrategias disciplinarias apropiadas para todo el grupo, en función de los estilos de aprendizaje, las carcterísticas de personalidad y la experiencia (Asensi, 2003).

\section{Convivencia escolar mediada por las TIC}

Hablar de convivencia escolar implica reconocer a un individuo que habita en compañía de otras personas y experimenta situaciones pacíficas, armónicas y de unión, pero también momentos de dificultades y crisis. En los espacios escolares, se refiere a todas aquellas relaciones en las que hay un objetivo común y un proceso de desarrollo y maduración progresivo (Garreton, 2014).

La convivencia escolar, implica el fortalecimiento de habilidades interpersonales, pero también, implica objetivos comunes en torno a comportamientos no violentos y de igualdad entre hombres y mujeres, lo cual se puede generar a través de talleres de prevención de la violencia escolar con perspectiva de género (Mendoza, 2015).
Es importante señalar que, convivencia y conflicto se presentan en un mismo escenario (Arrieta y Rojas, 2002). Esto se debe a que existen diferencias entre pensamientos, ideologías, actitudes y comportamientos (Herrera-Mendoza y Rico, 2014). Ahora bien, los medios de comunicación han venido presentando en los últimos años un sin número de noticias asociadas a la violencia escolar, generando una gran preocupación a nivel Nacional, por tal razón desde la academia se han propuesto diversas estrategias para mitigar dicha problemática (Caballero, 2010).

Sin embargo, la conflictividad escolar se ha convertido en una de las mayores preocupaciones de la sociedad, como se evidencia en los artículos aparecidos en los medios de comunicación en los últimos años (Caballero, 2010).

La convivencia se entiende como un medio de interacción social aprendido, a partir de las relaciones del ser humano con su entorno, implicando mecanismos de comunicación y respeto hacia los demás, por lo cual usualmente es necesario compartir criterios o ser tolerantes con las diferencias, para sostener una relación asertiva. Etimológicamente, el término convivencia proviene de conviviere, que significa vivir en compañía de otros, es decir, cohabitar. Convivir hace parte del reconocimiento personal con las que se debe compartir algún escenario o espacio sociales, sin embargo, estos sistemas están sujetos a normas que favorecen la interacción, para prevenir conflictos (López, Carvajal, Soto y Urrea, 2013).

Es así como surge, en la época moderna, la preocupación por una protección integral a los estudiantes, observándolos como sujetos potenciales en nuevas acciones de política legislativa en casi todos los países del mundo, lo que ha generado la 
consagración de los derechos, a través de preceptos, valores y principios constitucionales, generando un análisis científico. (Herrera, 2013).

Las TIC son tecnologías para almacenar, recuperar, procesar y comunicar la información. Existe una variedad de recursos electrónicos que se encuadran dentro del concepto de TIC como; televisión, teléfonos, videos, ordenadores, entre otros. Sin embargo, los medios más representativos de la sociedad actual son los computadores (Belloch, 2012).

Según Avendaño, Cortes \& Guerrero, (2015) expresan que el uso de las tecnologías de la información y la comunicación (TIC) tienen un papel importante en el desarrollo de competencias educativas y sociales, como factores predictores del desempeño académico en estudiantes de básica primaria. Coinciden en estas apreciaciones, autores como, Betancur et al (2014) y Marín et al (2017), cuando asocian las TIC a los procesos de mediación didáctica como herramientas clave o entornos virtuales que fortalecen procesos de interacción como base al desarrollo de competencias integrales en los estudiantes.

Por otro lado, Herrera, (2016) menciona que las TIC se han convertido en una herramienta innovadora en el contexto educativo generando una relación ineludible que se puede aplicar a cualquier modelo pedagógico a desarrollar y a tener presente de forma trasversal como instrumento pedagógico.

\section{Metodología}

La investigación es de tipo cualitativa, de alcance descriptivo y apoyada en los lineamientos de la IEP, en donde se busca articular las bases teóricas del estudio con la experiencia que viven los niños, niñas y adolescentes en la Insti- tución Educativa. (Mejía y Manjarrés, 2010)

\section{Participantes}

Durante el estudio participaron (178 estudiantes con edades que oscilaron entre los 6 y 17 años, distribuidos en los cursos de primaria y bachillerato de la Institución Educativa San José de Pueblo Viejo, del Departamento del Magdalena, Los estudiantes cumplieron con el diligenciamiento del consentimiento informado (tabla 1).

TABLA 1

Distribución de los participantes

\begin{tabular}{cc}
\hline Grado & $\begin{array}{c}\text { Número de } \\
\text { Estudiantes }\end{array}$ \\
\hline Primero & 12 \\
Segundo & 16 \\
Tercero & 25 \\
Cuarto & 13 \\
Quinto & 26 \\
Sexto & 38 \\
Séptimo & 12 \\
Octavo & 11 \\
Noveno & 25 \\
TOTAL & $\mathbf{1 7 8}$ \\
\hline
\end{tabular}

Fuente: elaboración propia, 2018.

\section{Técnicas e instrumentos}

Teniendo en cuenta que la IEP es una metodología libre, se seleccionaron técnicas e instrumentos de recolección de información propios de la investigación cualitativa, tales como, observación participante y bitácora del investigador, donde se consignaron todas las experiencias pedagógicas, observadas en el proceso de implementación de un programa disciplinario mediado por las TIC para mejorar la convivencia escolar. 


\section{Resultados}

Dentro de los principales hallazgos del estudio, se diseñó por parte de los estudiantes un programa disciplinario, con el acompañamiento de los docentes, donde se establecieron normas de convivencia en los carteles informativos, con la finalidad de mitigar los conflictos escolares, generando una solución pacífica de los mismos, el programa era claro frente a las sancio- nes pedagógicas, que estaban orientadas a trabajos comunitarios que favorecieran la institución y trabajos de investigación donde los jóvenes debían buscar temas relacionados con la problemática y encontrar la mejor solución para el mismo, con la finalidad que reflexionara sobre el hecho ocurrido, pero también tenía refuerzos positivos basados en la búsqueda de juegos virtuales que permitieran el aprendizaje de las diferentes asignaturas.

TABLA 2

Actividades pedagógicas para fortalecer los valores escolares

\begin{tabular}{l} 
Actividad \\
\hline Comités disciplinarios. \\
Reunión de los integrantes de \\
la investigación para discutir \\
los problemas que se están \\
presentando
\end{tabular}

Manejo de conflictos.

\section{Proyección de una Película.}

Juego el Tesoro de los Valores. Se esconde el tesoro que son los valores por el líder quien va dejando pistas realizando la búsqueda hasta que logren encontrar este tesoro el cual contiene una gran fortuna.

Aprender a escuchar.

Se hace un círculo en el aula iniciando con preguntas relacionadas a los valores.

Escogiendo varios tratando de que se repitan las intervenciones de los estudiantes respetando la palabra y escuchando cuando no es su turno.
Fortalecer el significado de los valores en los estudiantes.

Lograr que los estudiantes identifiquen el respeto, la generosidad y el trabajo en equipo.

generar espacios de respeto y de escucha.
Se refuerzan el respeto pues es fundamental la participación de cada uno y la escucha al momento de debatir el tema en específico.

Fuente: elaboración propia, 2018. 
De acuerdo a lo resultados se puede inferí que los estudiantes mejoraron la convivencia al interior del aula de clases, se fortalecieron valores convivenciales como el respeto, la armonía, la tolerancia a las diferencias entre otros. Las relaciones se afianzaron notándose en mayor medida en los grados en $2^{\circ}, 3^{\circ}$ y $5^{\circ}$, en los que el trabajo realizado fue de mayor impacto. Sin embargo, el proyecto debe seguirse desarrollando para buscar mejores estrategias y resultados, para luego implementarlo en otras Instituciones, lo que permitiría aumentar el grado de convivencia a nivel municipal.

\section{Discusión}

La implementación del programa disciplinario trajo consigo avances significativos en cuanto a la convivencia entre los estudiantes y la disminución de conflictos entre ellos. Con el desarrollo e las actividades programadas el trabajo en el aula de clases fue más ameno, participativo y de mayor motivación.

Así mismo los espacios de dialogo, la participación constante y las actividades programadas por el maestro se lograban iniciar y culminar de acuerdo a los objetivos planteados.

No se debe desconocer que para que en las aulas de clases no haya conflicto deben realizarse de manera constante actividades abiertas, libres y de gustos y preferencias por los estudiantes, fundamentado en las competencias ciudadanas, en normas de comportamientos, en premios y castigo de acuerdo a las acciones, en la resolución adecuada de conflicto, y sobre todo en la disposición práctica, metodológica y coherente por parte de los maestros.

El siguiente paso sería identificar otros mecanismos para ayudar a construir una institucionalidad escolar de corte disciplinario y moderno para garantizar un programa disciplinario eficiente (Amézquita, 2014)

\section{Referencias}

Amézquita, P. (2014). Minería y petróleo en Colombia: maldición interna de los recursos. Económicas CUC, 35(1), 4559.

Aquino, J. G. (1996). Indisciplina na escola. Alternativas Teóricas e Práticas. Grupo São Paulo: Editorial Summus.

Arrieta, M. y Rojas, A. (2002). Análisis de las garantías del proceso de alimentos y su eficacia dentro del proceso. [Tesis de pregrado]. Universidad del Norte, Barranquilla, Colombia.

Avendaño, I., Cortés, O. y Guerrero, H. (2015). Competencias sociales y tecnologías de la información y la comunicación como factores asociados al desempeño en estudiantes de básica primaria con experiencia de desplazamiento forzado. Diversitas, 11(1), 13-36.

Belloch. (2012). Las Tecnologías de la Información y Comunicación en el aprendizaje. Universidad de Valencia. Recuperado de https://www.uv.es/bellochc/pedagogia/EVA1.pdf

Betancur, S., Carmona, L., Contreras, R., Karam, J., Maestre, N., Romero, Y. y Uribe, S. (2014). Videojuegos y tic como Estrategias Pedagógicas: Formación para el uso seguro de internet. Cultura Educación y Sociedad, 5(1). Recuperado de https://revistascientificas.cuc. edu.co/culturaeducacionysociedad/article/view/997

Caballero, M. (2010). Convivencia escolar. Un estudio sobre buenas prácticas. $R e$ vista Paz y Conflictos. 3. 154-169.

Castro, L. y Fernández, E. (2003). Indisciplina y violencia escolar. En, III Jornadas de Sociología de la UNLP, La Argentina de la crisis: Recomposición, nuevos actores y el rol de los intelectuales. Universidad Nacional de la Plata. La Plata, Argentina 
De Bustillo, M., Pérez, D. y Martín, E. (2006). ¿Qué penalizan los docentes? análisis de la disciplina a través de los partes de incidencia. Infancia y Aprendizaje, 29(4), 423-435.

Del Rey, R., Ortega, R. y Feria, I. (2009). Convivencia escolar: fortaleza de la comunidad educativa y protección ante la conflictividad escolar. Revista interuniversitaria de formación del profesorado, 23(3).

González, J., Moreno, L. y Rodríguez, M. (2015). La integración y sus convenios internacionales en América Latina. Económicas CUC, 36(2), 79-94.

Hernández, M. y Solano, I. (2007). Ciberbullying, un problema de acoso escolar. RIED. Revista iberoamericana de educación a distancia, 10(1).

Herrera, J. y Domínguez, K. (2009). Factores de riesgo asociados a indisciplina $\mathrm{y}$ aprovechamiento escolar en alumnos de 60 año de primaria. En, X Congreso Nacional de Investigación Educativa. Veracruz, México

Herrera-Mendoza, K., y Rico, R. (2014). El Clima Escolar Como Elemento Fundamental de la Convivencia en la Escuela. Revista escenarios. 12(2). 7-18.

Herrera-Tapias, B. (2016). Cultura Ciudadana y las Tecnologías de la Información y la Comunicación. Barranquilla: YOYOBIZ.
Herrera-Tapias, B. (2013). La constitucionalización de los derechos del consumidor en Colombia: un análisis desde los derechos sociales fundamentales. Civilizar. Ciencias Sociales y Humanas, 13 (25), 33-47.

Kadi, O. y Acevedo, Á. (2014). Liderazgo ético frente a la diversidad cultural dentro de las organizaciones con régimen disciplinario. Económicas CUC, 35(2), 75-88.

López C, Carvajal C., Soto M. y Urrea P. (2013). Factores asociados a la convivencia escolar en adolescentes. Rev. Educación y Educadores, 16(3). 383-410.

Marín, F., Niebles, M., Sarmiento, M. y Valbuena, S. (2017): Mediación de las tecnologías de la información en la comprensión lectora para la resolución de problemas aritméticos de enunciado verbal. Espacios, 38(20). 13-20. Recuperado de http://www.revistaespacios. com/a17v38n20/a17v38n20p20.pdf

Mendoza, A. (2015). Propuesta de convivencia escolar a través de talleres de prevención de violencia escolar con perspectiva de género. Cultura Educación y Sociedad, 6(2).

Narodowski, M. (2012). El Bullying Como Sustituto De La Vieja Indisciplina: estrategias frente a la disolución de la autoridad adulta. Revista Linhas, 13(2), 140-151. 